\title{
OSCULATION BY ALGEBRAIC HYPERSURFACES
}

\author{
JAY A. WOOD
}

\section{Introduction}

In this paper we give necessary and sufficient conditions for $d$ pieces of hypersurface to be osculated to a fixed order by an algebraic hypersurface of degree $d$.

Given a line $L_{0}$ in $P^{n+1}$ and $d$ points $P_{1}^{0}, \cdots, P_{d}^{0}$ on $L_{0}$, suppose there are $d$ pieces of hypersurface $\gamma_{1}, \cdots, \gamma_{d}$ such that $P_{i}^{0} \in \gamma_{i}$ and $L_{0}$ intersects each $\gamma_{i}$ transversely.

The question addressed here is: when does there exist an algebraic hypersurface $\gamma$ of degree $d$ which osculates each piece $\gamma_{i}$ to order $r$ at $P_{i}^{0}$ ? The main result gives necessary and sufficient conditions for the existence of such an algebraic hypersurface $\gamma$.

Fix affine coordinates $\left(x_{0}, \cdots, x_{n}\right)$ on $P^{n+1}$, and fix line coordinates $\left(m_{1}, \cdots, m_{n}, b_{1}, \cdots, b_{n}\right)$, where a line $L$ is given by $x_{k}=m_{k} x_{0}+b_{k}, k=$ $1,2, \cdots, n$. (Line coordinates are just local coordinates on $\operatorname{Gr}(1, n+1)$, the Grassmannian of all lines in $P^{n+1}$.) Assume that coordinates have been chosen so that the given line $L_{0}$ has line coordinates $m_{k}=0, b_{k}=0$ for all $k$. $L_{0}$ is then the $x_{0}$-axis. For convenience, write $m=\left(m_{1}, \cdots, m_{n}\right), b=\left(b_{1}, \cdots, b_{n}\right)$. A line $L=L(m, b)$ near $L_{0}$ will intersect each $\gamma_{i}$ at a point $P_{i}=P_{i}(m, b)$. Then $P_{i}(0,0)=P_{i}^{0}$. Let $X_{i}=X_{i}(m, b)$ be the 0 th coordinate of $P_{i}$ in terms of the affine coordinate system. Define $K_{j k}=K_{j k}(m, b)$ by

$$
K_{j k}(m, b)=\frac{\partial^{2}\left[\Sigma_{i} X_{i}(m, b)\right]}{\partial b_{j} \partial b_{k}}
$$

$j, k=1,2, \cdots, n$. We can now state the main result.

Theorem. There exists an algebraic hypersurface $\gamma$ of degree $d$, which osculates each $\gamma_{i}$ to order $r, 2 \leqslant r \leqslant d$, at $P_{i}^{0}, i=1,2, \cdots, d$, if and only if $K_{j k}$ and all of its partial derivatives of order $\leqslant r-2$ vanish at $(m, b)=(0,0)$.

Remarks. 1. If the order of osculation desired is $r=0$ or $r=1$, there is no condition. Just take $\gamma$ to be the union of the $d$ tangent hyperplanes to $\gamma_{i}$ at $P_{i}^{0}$. 
2. In order to handle the case $r \geqslant d$, one must make assumptions on the derivatives of the individual $X_{i}$ 's not just on their sum. The needed conditions are obvious, and we will not concern ourselves with them.

3. The case of second-order osculations for curves in the plane $(n=1, r=2)$ is treated by Griffiths-Harris [1, pp. 698-699]. The single condition obtained in that case is called the Reiss relation. The conditions of the theorem above may be considered as generalized Reiss relations.

Proof. The proof is in two parts. Each part will be treated in a separate section below. The necessity of the conditions follows from a direct computation along the lines of some earlier work of the author [3]. The sufficiency of the conditions will follow from a cohomological argument which counts the number of obstructions to the existence of $\gamma$. This cohomology argument is a direct generalization of the treatment by Griffiths-Harris of the $n=1, r=2$ case, as mentioned above.

Necessity. Suppose there exists an algebraic hypersurface $\gamma$ of degree $d$, which osculates each piece of hypersurface $\gamma_{i}$ to order $r$ at $P_{i}^{0}$. In addition, suppose that $\gamma$ has defining equation

$$
p\left(x_{0}, \cdots, x_{n}\right)=0,
$$

where $p$ is a polynomial degree $d$, and that $\gamma_{i}$ has local defining equation

$$
\phi_{i}\left(x_{0}, \cdots, x_{n}\right)=0 \text {. }
$$

Because $L_{0}\left(=x_{0}\right.$-axis) meets $\gamma$ and each $\gamma_{i}$ transversely, the above equations define $x_{0}$ implicitly as a function of $x_{1}, \cdots, x_{n}$ locally near each $P_{i}^{0}$; say, for $\gamma$ near $P_{i}^{0}$,

$$
x_{0}=\pi_{i}\left(x_{1}, \cdots, x_{n}\right) \quad\left(\text { defining } \pi_{i}\right),
$$

and for $\gamma_{i}$ near $P_{i}^{0}$,

$$
x_{0}=\Phi_{i}\left(x_{1}, \cdots, x_{n}\right) \quad\left(\text { defining } \Phi_{i}\right) .
$$

Remember that our choice of coordinates dictates that $\pi_{i}(0, \cdots, 0)=$ $\Phi_{i}(0, \cdots, 0)=X_{i}(0,0)$. Osculation to order $r$ now means that the function $\pi_{i}-\Phi_{i}$ vanishes through order $r$ at $\left(x_{1}, \cdots, x_{n}\right)=(0, \cdots, 0)$. Recall that $X_{i}(m, b)$ is the 0th coordinate of the point of intersection of $\gamma_{i}$ and $L(m, b)$. Now define $Y_{i}=Y_{i}(m, b)$ to be the 0 th coordinate of the point of intersection of $\gamma$ and $L(m, b)$ near the point of intersection of $\gamma_{i}$ and $L(m, b)$. Because $\gamma$ osculates $\gamma_{i}, Y_{i}$ will be close to $X_{i}$ for $(m, b)$ near $(0,0)$. More precisely, we have the following lemma.

Lemma. If $\gamma$ osculates $\gamma_{i}$ to order $r$ at $P_{i}^{0}$, then the function $X_{i}(m, b)-$ $Y_{i}(m, b)$ vanishes up through order $r$ at $(m, b)=(0,0)$.

Proof. $X_{i}-Y_{i}$ vanishes (to order zero) at $(0,0)$ because $X_{i}(0,0)=$ $\Phi_{i}(0, \cdots, 0), Y_{i}(0,0)=\pi_{i}(0, \cdots, 0)$, and $\Phi_{i}(0, \cdots, 0)=\pi_{i}(0, \cdots, 0)$. 
Before proceeding to the higher orders, observe that $X_{i}(m, b)=$ $\Phi_{i}\left(m X_{i}(m, b)+b\right)$, where $m X_{i}(m, b)+b$ is short-hand for $\left(m_{1} X_{i}(m, b)+\right.$ $\left.b_{1}, \cdots, m_{n} X_{i}(m, b)+b_{n}\right)$. Similarly, $Y_{i}(m, b)=\pi_{i}\left(m Y_{i}(m, b)+b\right)$.

Let $\partial_{m}^{J}, \partial_{b}^{K}$ be multi-indexed partial derivatives with respect to the $m$ - and $b$-variables respectively.

As an induction hypothesis, suppose $X_{i}-Y_{i}$ vanishes up through order $s$ $(<r)$ at $(0,0)$. Consider one of the $(s+1)$ st order partial derivatives $\partial\left[\partial_{m}^{J} \partial_{b}^{K}\left(X_{i}-Y_{i}\right)\right] / \partial m_{1}$, where $|J|+|K|=s$.

$$
\begin{aligned}
\frac{\partial}{\partial m_{l}}[ & \left.\partial_{m}^{J} \partial_{b}^{K}\left(X_{i}-Y_{i}\right)\right] \\
& =\partial_{m}^{J} \partial_{b}^{K}\left[\frac{\partial}{\partial m_{l}}\left(\Phi_{i}\left(m X_{i}(m, b)+b\right)\right)-\frac{\partial}{\partial m_{l}}\left(\pi_{i}\left(m Y_{i}(m, b)+b\right)\right)\right] \\
& =\partial_{m}^{J} \partial_{b}^{K}\left[\sum_{j} \frac{\partial \Phi_{i}}{\partial x_{j}}\left(\delta_{j l} X_{i}+m_{j} \frac{\partial X_{i}}{\partial m_{l}}\right)-\sum_{j} \frac{\partial \pi_{i}}{\partial x_{j}}\left(\delta_{j l}+m_{j} \frac{\partial Y_{i}}{\partial m_{l}}\right)\right],
\end{aligned}
$$

where $\partial \Phi_{i} / \partial x_{j}$ and $\partial \pi_{i} / \partial x_{j}$ are evaluated at $m X_{i}+b$ and $m Y_{i}+b$ respectively.

The remaining derivatives remain to be calculated, but are easily visualized. Because of the product rule, any $(s+1)$ st order derivative of either $X_{i}$ or $Y_{i}$ is multiplied by an $m_{j}$. After evaluating the expression at $(m, b)=(0,0)$, such terms vanish. The remaining terms are expressible in terms of derivatives of $\Phi_{i}$ and $\pi_{i}$ to orders $\leqslant s+1 \leqslant r$ and derivatives of $X_{i}$ and $Y_{i}$ to orders $\leqslant s$. Moreover, the entire expression is clearly skew-symmetric in the pairs $\left(\Phi_{i}, X_{i}\right)$ and $\left(\pi_{i}, Y_{i}\right)$. When we evaluate at $(m, b)=(0,0)$, the $\Phi_{i}, \pi_{i}$ terms are evaluated at $m X_{i}$ [or $Y_{i}$ ] $+b=0$. $\Phi_{i}-\pi_{i}$ vanishes to order $r$ at $(0, \cdots, 0)$ by the osculation assumption, and $X_{i}-Y_{i}$ vanishes through order $s$ at $(0,0)$ by induction. Thus the expression is also symmetric in the pairs $\left(\Phi_{i}, X_{i}\right)$ and $\left(\pi_{i}, Y_{i}\right)$, and consequently vanishes.

A similar argument holds for $\partial\left[\partial_{m}^{J} \partial_{b}^{K}\left(X_{i}-Y_{i}\right)\right] / \partial b_{l}$.

The necessity of the conditions in the main theorem then follows from the following theorem which was first proved in [3]. Since its proof is very simple, we include it here for convenience.

Theorem. If $\gamma$ is an algebraic hypersurface in $P^{n+1}$ of degree $d$, and if $Y_{i}(m, b)(i=1, \cdots, d)$ are the 0 th coordinates of the intersection points of $\gamma$ with a line $L(m, b)$, then

$$
\frac{\partial^{2}}{\partial b_{j} \partial b_{k}} \sum_{i} Y_{i}(m, b)=0
$$

for all $(m, b)$, and for all $j, k=1, \cdots, n$. 
Proof. If $\gamma$ satisfies the polynomial equation of degree $d$ :

$$
p\left(x_{0}, \cdots, x_{n}\right)=0 \text {, }
$$

then the $Y_{i}$ 's are the $d$ solutions of the following equation (in $x_{0}$ ):

$$
\tilde{p}\left(x_{0}\right)=p\left(x_{0}, m_{1} x_{0}+b_{1}, \cdots, m_{n} x_{0}+b_{n}\right)=0 .
$$

$\tilde{p}$ is a polynomial of degree $d$ in $x_{0}$, whose coefficients are in turn polynomials in $(m, b)$. If we set

$$
\tilde{p}\left(x_{0}\right)=A_{0}(m, b) x_{0}^{d}-A_{1}(m, b) x_{0}^{d-1}+\cdots \pm A_{d}(m, b),
$$

then the definition of $\tilde{p}$ in terms of $p$ implies that $A_{0}$ is independent of $b$ and that $A_{1}$ is linear in $b$.

It is well known that the sum of the roots of $\tilde{p}$, namely $\Sigma Y_{i}(m, b)$, is expressible in terms of the coefficients of $\tilde{p}$. Specifically,

$$
\sum Y_{i}(m, b)=A_{1} / A_{0} \text {. }
$$

Since $A_{1} / A_{0}$ is only linear in $b$, the second $b$-partials must vanish.

Sufficiency. To prove sufficiency, we generalize the approach in GriffithsHarris [1, pp. 698-699]. The author would like to thank Phillip Griffiths, Lawrence Ein and Raghavan Narasimhan for their helpful comments and advice.

Henceforth we shall denote $P^{n+1}$ simply by $P$. Let $I$ be the ideal sheaf of the line $L_{0}$ in $P$. Let $O$ be the structure sheaf of $P$, and let $O_{s}=O / I^{s+1}$. In particular, $O_{0}$ is the structure sheaf of $L_{0}$. Define the sth infinitesimal neighborhood of $L_{0}$ to be the scheme $L_{s}=\left(L_{0}, O_{s}\right)$. Let $\operatorname{Pic}(V)$ denote the set of Cartier divisors on a scheme $V$, modulo linear equivalence. $\operatorname{Pic}(V)$ is isomorphic to $H^{1}\left(V, O_{V}^{*}\right)$.

An $s$ th order germ of hypersurface intersecting $L_{0}$ defines an element $\eta$ of $\operatorname{Pic}\left(L_{s}\right)$, together with a section of $\eta$. There is also a natural restriction map $a_{s}: \operatorname{Pic}(P) \rightarrow \operatorname{Pic}\left(L_{s}\right)$ obtained by intersection with $L_{s}$. Our problem of determining when there exists an algebraic hypersurface $\gamma$ which osculates $\gamma_{i}$ to $r$ th order at $P_{i}^{0}$ is equivalent to first determining when an element $D \in \operatorname{Pic}\left(L_{r}\right)$ is in the image of the map $a_{r}: \operatorname{Pic}(P) \rightarrow \operatorname{Pic}\left(L_{r}\right)$, and then determining whether sections of $D$ lift.

Using $\operatorname{Pic}(P) \cong H^{1}\left(P, O^{*}\right)$ and $\operatorname{Pic}\left(L_{r}\right) \cong H^{1}\left(L_{r}, O_{r}^{*}\right)$, information about the image of $a_{r}: H^{1}\left(P, O^{*}\right) \rightarrow H^{1}\left(L_{r}, O_{r}^{*}\right)$ can be obtained in the following manner. From the exact sequence

$$
0 \rightarrow I^{r+1} \rightarrow O \rightarrow O_{r} \rightarrow 0,
$$

exponentiate to obtain the multiplicativve version

$$
0 \rightarrow J_{r+1} \rightarrow O^{*} \rightarrow O_{r}^{*} \rightarrow 0 \text {. }
$$


The long exact sequence on cohomology includes

$$
\cdots \rightarrow H^{1}\left(P, O^{*}\right) \rightarrow H^{1}\left(L_{r}, O_{r}^{*}\right) \rightarrow H^{2}\left(P, J_{r+1}\right) \rightarrow H^{2}\left(P, O^{*}\right) \rightarrow \cdots
$$

It is easy to see that $H^{2}\left(P, O^{*}\right)=0$. Indeed, from the exact exponential sheaf sequence

$$
0 \rightarrow Z \rightarrow O \rightarrow O^{*} \rightarrow 0,
$$

follows the long exact cohomology sequence

$$
\cdots \rightarrow H^{2}(P, O) \rightarrow H^{2}\left(P, O^{*}\right) \rightarrow H^{3}(P, Z) \rightarrow \cdots .
$$

But $H^{2}(P, O)=0$ and $H^{3}(P, Z)=0$, so that $H^{2}\left(P, O^{*}\right)=0$ also. Thus sequence $(*)$ above becomes

$$
\cdots \rightarrow H^{1}\left(P, O^{*}\right) \rightarrow H^{1}\left(L_{r}, O_{r}^{*}\right) \rightarrow H^{2}\left(P, J_{r+1}\right) \rightarrow 0,
$$

where the first map is $a_{r}$. Thus the obstructions to $D \in H^{1}\left(L_{r}, O_{r}^{*}\right)$ being in the image of $a_{r}$ lie in $H^{2}\left(P, J_{r+1}\right)$. Since $J_{r+1} \cong I^{r+1}$, we will next calculate $h^{2}\left(P, I^{r+1}\right)$.

Let $N^{*}=I / I^{2}$ be the conormal bundle of $L_{0}$ in $P$. Because $L_{0}$ is just a line in $P, N^{*} \cong O_{0}^{n}(-1)$ where the superscript $n$ means to take the direct sum of $n$ copies of $O_{0}(-1)$.

Proposition. $H^{i}\left(P^{n+1}, I\right)=H^{i}\left(P^{n+1}, I^{2}\right)=0$ for $i \geqslant 0$. For $r \geqslant 2$, $H^{i}\left(P^{n+1}, I^{r+1}\right)=0, i \neq 2$, and

$$
h^{2}\left(P^{n+1}, I^{r+1}\right)=\left(\begin{array}{c}
n+1 \\
2
\end{array}\right)+2\left(\begin{array}{c}
n+2 \\
3
\end{array}\right)+\cdots+(r-1)\left(\begin{array}{c}
n-1+r \\
r
\end{array}\right) .
$$

Proof. First, from the definition of $I$ we have the exact sheaf sequence $0 \rightarrow I \rightarrow O \rightarrow O_{0} \rightarrow 0$, where, as above, $O$ is the structure sheaf of $P=P^{n+1}$, and $O_{0}$ is the structure sheaf of the line $L_{0}$. On cohomology we have

$$
\begin{aligned}
0 & \rightarrow H^{0}(P, I) \rightarrow H^{0}(P, O) \rightarrow H^{0}\left(L_{0}, O_{0}\right) \\
& \rightarrow H^{1}(P, I) \rightarrow H^{1}(P, O) \rightarrow H^{1}\left(L_{0}, O_{0}\right) \rightarrow \cdots .
\end{aligned}
$$

But $H^{0}(P, O) \cong H^{0}\left(L_{0}, O_{0}\right) \cong\{$ constants $\}$, and $H^{i}(P, O) \cong H^{i}\left(L_{0}, O_{0}\right)=0$ for $i \geqslant 1$, so that $H^{i}(P, I)=0$ for all $i \geqslant 0$.

Second, note the exact sequence $0 \rightarrow I^{2} \rightarrow I \rightarrow N^{*} \rightarrow 0$, which induces the long exact sequence

$$
\cdots \rightarrow H^{i-1}\left(L_{0}, N^{*}\right) \rightarrow H^{i}\left(P, I^{2}\right) \rightarrow H^{i}(P, I) \rightarrow H^{i}\left(L_{0}, N^{*}\right) \rightarrow \cdots .
$$

Because $H^{i}\left(L_{0}, O_{0}(-1)\right)=0$ for all $i \geqslant 0$, we see that $H^{i}(P, I) \cong H^{i}\left(P, I^{2}\right)$. So $H^{i}\left(P, I^{2}\right)=0$ for all $i \geqslant 0$.

We prove the remainder of the proposition by induction on $r$. For the case $r=2$ we use that

$$
I^{2} / I^{3} \cong \operatorname{Sym}^{2}\left(N^{*}\right) \cong O_{0}^{\mathrm{rk}(2)}(-2)
$$


where $\operatorname{rk}(2)=\operatorname{rank}\left(\operatorname{Sym}^{2}\left(N^{*}\right)\right)$, namely, $\left(\begin{array}{c}n+1 \\ 2\end{array}\right)$. Then

$$
0 \rightarrow I^{3} \rightarrow I^{2} \rightarrow \operatorname{Sym}^{2}\left(N^{*}\right) \rightarrow 0
$$

induces

$$
\begin{aligned}
\cdots & \rightarrow H^{1}\left(P, I^{2}\right) \rightarrow H^{1}\left(L_{0}, \operatorname{Sym}^{2}\left(N^{*}\right)\right) \rightarrow H^{2}\left(P, I^{3}\right) \\
& \rightarrow H^{2}\left(P, I^{2}\right) \rightarrow H^{2}\left(L_{0}, \operatorname{Sym}^{2}\left(N^{*}\right)\right) \rightarrow \cdots .
\end{aligned}
$$

Because $H^{i}\left(P, I^{2}\right)=0$ for all $i \geqslant 0$, and $H^{i}\left(L_{0}, O_{0}(-2)\right)=0$ for $i \neq 1$, we see that $H^{2}\left(P, I^{3}\right) \cong H^{1}\left(L_{0}, \operatorname{Sym}^{2}\left(N^{*}\right)\right)$ and that $H^{i}\left(P, I^{3}\right)=0$ for $i \neq 2$. By Serre duality, $h^{1}\left(L_{0}, O_{0}(-2)\right)=h^{0}\left(L_{0}, O_{0}\right)=1$. Thus $h^{2}\left(P, I^{3}\right)=1 \cdot \operatorname{rk}(2)=$ $\left(\begin{array}{c}n+1 \\ 2\end{array}\right)$.

For the induction step, we assume the result for $r=s$ and prove it for $r=s+1$. Using the isomorphism

$$
I^{s} / I^{s+1} \cong \operatorname{Sym}^{s}\left(N^{*}\right) \cong O_{0}^{\mathrm{rk}(s)}(-s),
$$

where $\operatorname{rk}(s)=\operatorname{rank}\left(\operatorname{Sym}^{s}\left(N^{*}\right)\right)=\left(\begin{array}{c}n-1-s \\ s\end{array}\right)$, we get the exact sheaf sequence

$$
0 \rightarrow I^{s+1} \rightarrow I^{s} \rightarrow \operatorname{Sym}^{s}\left(N^{*}\right) \rightarrow 0 .
$$

The long exact cohomology sequence contains

$$
\begin{aligned}
\cdots & \rightarrow H^{1}\left(P, I^{s}\right) \rightarrow H^{1}\left(L_{0}, \operatorname{Sym}^{s}\left(N^{*}\right)\right) \rightarrow H^{2}\left(P, I^{s+1}\right) \\
& \rightarrow H^{2}\left(P, I^{s}\right) \rightarrow H^{2}\left(L_{0}, \operatorname{Sym}^{s}\left(N^{*}\right)\right) \rightarrow \cdots .
\end{aligned}
$$

Because $h^{1}\left(L_{0}, O_{0}(-s)\right)=h^{0}\left(L_{0}, O_{0}(s-2)\right)=s-1$, and all other $h^{i}\left(L_{0}\right.$, $\left.O_{0}(-s)\right)=0$ for $i \neq 1$, the induction hypothesis on $h^{i}\left(P, I^{s}\right)$ forces $h^{i}\left(P, I^{s+1}\right)$ $=0$ for $i \neq 2$, and the long exact sequence collapses to

$$
0 \rightarrow H^{1}\left(L_{0}, \operatorname{Sym}^{s}\left(N^{*}\right)\right) \rightarrow H^{2}\left(P, I^{s+1}\right) \rightarrow H^{2}\left(P, I^{s}\right) \rightarrow 0 .
$$

Then

$$
\begin{aligned}
& h^{2}\left(P, I^{s+1}\right)=h^{2}\left(P, I^{s}\right)+h^{1}\left(L_{0}, \operatorname{Sym}^{s}\left(N^{*}\right)\right) \\
& =h^{2}\left(P, I^{s}\right)+(s-1) \operatorname{rk}(s)=h^{2}\left(P, I^{s}\right)+(s-1)\left({ }_{s}^{1+s}\right),
\end{aligned}
$$

and the induction step is complete.

We conclude the first part of the proof of sufficiency by showing that the number of independent conditions among the necessary conditions (i.e., that $K_{j k}$ and its partial derivatives through order $r-2$ vanish at $\left.(m, b)=0\right)$ is exactly equal to the dimension of the space of obstructions $H^{2}\left(P, I^{r+1}\right)$.

For $r=2, h^{2}\left(P, I^{3}\right)=\left(\begin{array}{c}n+1 \\ 2\end{array}\right)$. On the other hand $K_{j k}=K_{k j}$, so that the number of independent components is also $\left(\begin{array}{c}n+1 \\ 2\end{array}\right)$. 
By induction, it suffices to prove that the number of independent partial derivatives of $K_{j k}$ of order exactly $s-2$ is equal to

$$
h^{2}\left(P, I^{s+1}\right)-h^{2}\left(P, I^{s}\right)=h^{1}\left(L_{0}, \operatorname{Sym}^{s}\left(N^{*}\right)\right)=(s-1)\left(\begin{array}{c}
n-1+s \\
s
\end{array}\right) .
$$

At first glance, this appears hopeless because even in the case $s=3$, the first-order partial derivatives of $K_{j k}$ are

$$
\begin{aligned}
\partial\left[K_{j k}\right] / \partial m_{l} & =\partial^{3}\left[\sum X_{i}\right] / \partial b_{j} \partial b_{k} \partial m_{l}, \\
\partial\left[K_{j k}\right] / \partial b_{l} & =\partial^{3}\left[\sum X_{i}\right] / \partial b_{j} \partial b_{k} \partial b_{l},
\end{aligned}
$$

of which there appear to be $\left(\begin{array}{c}n+2 \\ 3\end{array}\right)+n\left(\begin{array}{c}n+1 \\ 2\end{array}\right)$ which are independent.

Fortunately, there are relations among the partial derivatives of $\Sigma X_{i}$.

Recall that $X_{i}(m, b)$ is the 0th coordinate of the point of intersection of $L(m, b)$ with $\gamma_{i}$, that $\gamma_{i}$ has defining equation $\phi_{i}\left(x_{0}, \cdots, x_{n}\right)=0$ and that $L(m, b)$ satisfies $x_{k}=m_{k} x_{0}+b_{k}$ for $k=1, \cdots, n$. Thus $X_{i}$ satisfies the identity

$$
\phi_{i}\left(X_{i}, m_{1} X_{i}+b_{1}, \cdots, m_{n} X_{i}+b_{n}\right)=0,
$$

for all $(m, b)$. Differentiation of this identity with respect to $m_{j}$ yields

$$
\left(\partial \phi_{i} / \partial x_{0}\right)\left(\partial X_{i} / \partial m_{j}\right)+\sum_{k}\left[\left(\partial \phi_{i} / \partial x_{k}\right)\left(m_{k} \partial X_{i} / \partial m_{j}+\delta_{k j} X_{i}\right)\right]=0,
$$

while differentiation with respect to $b_{j}$ yields

$$
\left(\partial \phi_{i} / \partial x_{0}\right)\left(\partial X_{i} / \partial b_{j}\right)+\sum_{k}\left[\left(\partial \phi_{i} / \partial x_{k}\right)\left(m_{k} \partial X_{i} / \partial b_{j}+\delta_{k j}\right)\right]=0 .
$$

From these two equations it follows that

$$
\partial X_{i} / \partial m_{j}=X_{i}\left(\partial X_{i} / \partial b_{j}\right) \text {. }
$$

Equipped with this relation, let us reexamine the case $s=3$ :

$$
\begin{aligned}
\frac{\partial\left[K_{j k}\right]}{\partial m_{l}}= & \frac{\partial^{3}\left[\sum X_{i}\right]}{\partial b_{j} \partial b_{k} \partial m_{l}}=\frac{\partial^{2}\left[\sum X_{i}\left(\partial X_{i} / \partial b_{l}\right)\right]}{\partial b_{j} \partial b_{k}} \\
& =\frac{\partial}{\partial b_{j}}\left(\sum \frac{\partial X_{i}}{\partial b_{k}} \frac{\partial X_{i}}{\partial b_{l}}+\sum X_{i} \frac{\partial^{2} X_{i}}{\partial b_{k} \partial b_{l}}\right) \\
& =\sum X_{i} \frac{\partial^{3} X_{i}}{\partial b_{j} \partial b_{k} \partial b_{l}}+\sum\left(\frac{\partial X_{i}}{\partial b_{j}} \frac{\partial^{2} X_{i}}{\partial b_{k} \partial b_{l}}+\frac{\partial X_{i}}{\partial b_{k}} \frac{\partial^{2} X_{i}}{\partial b_{j} \partial b_{l}}+\frac{\partial X_{i}}{\partial b_{l}} \frac{\partial^{2} X_{i}}{\partial b_{j} \partial b_{k}}\right), \\
& \frac{\partial\left[K_{j k}\right]}{\partial b_{l}}=\frac{\sum \partial^{3} X_{i}}{\partial b_{j} \partial b_{k} \partial b_{l}} .
\end{aligned}
$$


Now it is clear that there are only $2\left({ }_{3}^{n-1+3}\right)=h^{1}\left(L_{0}, \operatorname{Sym}^{s}\left(N^{*}\right)\right)$ independent components among the first partial derivatives of $K_{j k}$. The form of the general derivative of $K_{j k}$ of order $s$ is now predictable.

Proposition. Let $E$ and $F$ be multi-indices with $|E|=e$ and $|F|=f$. Let $G=E+F$. If $e+f=s$, then

$$
\partial^{s}\left[K_{j k}\right] / \partial m^{E} \partial b^{F} \equiv \sum X_{i}^{e}\left(\partial^{s+2} X_{i} / \partial b^{G} \partial b_{j} \partial b_{k}\right),
$$

modulo terms containing derivatives of $X_{i}$ of order $1, \cdots, s-1$.

Proof. For $s=0,1$, this has already been shown above. The induction step is quite easy. If $e+f=s$, then

$$
\begin{aligned}
\frac{\partial^{s+1}\left[K_{j k}\right]}{\partial m_{l} \partial m^{E} \partial b^{F}} & \equiv \frac{\partial}{\partial m_{l}}\left[\sum X_{i}^{e} \frac{\partial^{s+2} X_{i}}{\partial b^{G} \partial b_{j} \partial b_{k}}\right] \\
& \equiv\left\{\sum e X_{i}^{e-1} X_{i} \frac{\partial X_{i}}{\partial b_{l}} \frac{\partial^{s+2} X_{i}}{\partial b^{G} \partial b_{j} \partial b_{k}}\right\}+\sum X_{i}^{e} \frac{\partial^{s+2}\left[X_{i} \partial X_{i} / \partial b_{l}\right]}{\partial b^{G} \partial b_{j} \partial b_{k}} \\
& \equiv \sum X_{i}^{e+1} \frac{\partial^{s+3} X_{i}}{\partial b^{G} \partial b_{j} \partial b_{k} \partial b_{l}}
\end{aligned}
$$

modulo derivatives of order $\leqslant s$.

A similar computation holds for $\partial^{s+1}\left[K_{j k}\right] / \partial b_{l} \partial m^{E} \partial b^{F}$.

We next note that

$$
\frac{\partial^{2} X_{i}}{\partial m_{j} \partial b_{k}}=\frac{\partial}{\partial b_{k}}\left[\frac{X_{i} \partial X_{i}}{\partial b_{j}}\right]=X_{i} \frac{\partial^{2} X_{i}}{\partial b_{j} \partial b_{k}}+\frac{\partial X_{i}}{\partial b_{j}} \frac{\partial X_{i}}{\partial b_{k}}
$$

is symmetric in $j$ and $k$. Thus the expression for $\partial^{s}\left[K_{j k}\right] / \partial m^{E} \partial b^{F}$, once expressed entirely in terms of $b$-derivatives of $X_{i}$, is a symmetric expression in the $s+2$ indices which appear. However, the highest order terms are distinguished by the power of $X_{i}$ appearing as a factor. That power is precisely $e$. Because $e$ can be any number from 0 to $s$, there will be $s+1$ independent groups of derivatives with each group in turn depending upon $s+2$ symmetric indices. Thus the total number of independent components among the derivatives of $K_{j k}$ of order $s$ is $(s+1)\left(\begin{array}{c}n-1+s+2 \\ s+2\end{array}\right)$. For $s=r-2$, the number of independent components is $(r-1)\left({ }_{r}^{n-1+r}\right)$ which is precisely $h^{1}\left(L_{0}, \operatorname{Sym}^{\mathrm{s}}\left(N^{*}\right)\right)$.

The number of independent necessary conditions being equal to the number of obstructions to $D \in \operatorname{Pic}\left(L_{r}\right)$ being in the image of $a_{r}: \operatorname{Pic}(P) \rightarrow \operatorname{Pic}\left(L_{r}\right)$, the necessary conditions are also sufficient. This concludes the first part of the proof of sufficiency.

The second part of the proof of sufficiency is concerned with lifting sections. Our original data of $d$ pieces of hypersurface meeting $L_{0}$ determine both a line 
bundle $D \in \operatorname{Pic}\left(L_{r}\right)$ and a section $\sigma$ of $D$. Assuming the conditions stated in the theorem we have just proved the existence of a line bundle $E$ on $P^{n+1}$ such that $E$ restricts to $D$. To get an actual hypersurface $\gamma$ which osculates the original $\gamma_{i}$ 's, we must lift the section $\sigma$ of $D$ to a section $\tau$ of $E$.

Because there were $d$ pieces of hypersurface and $E$ restricts to a divisor linearly equivalent to $D$, the degree of $E$ is also $d$. Thus $E \cong O(d)$ on $P^{n+1}$. The restriction map $E \rightarrow D \rightarrow 0$ has a kernel consisting of elements of $E \cong O(d)$ which vanish on $L_{r}$, namely $I^{r+1}(d)$. To prove that a section $\sigma$ of $D$ can be lifted to a section $\tau$ of $E$, it suffices to prove that $H^{1}\left(P^{n+1}, I^{r+1}(d)\right)=0$. This will follow from the following proposition.

Proposition. If $s \geqslant 1$ and $d \geqslant s-2$, then $H^{i}\left(P^{n+1}, I^{s}(d)\right)=0$, for $i \geqslant 1$.

Proof. If $P^{n+1}$ has homogeneous coordinates $\left[x_{0}, \cdots, x_{n+1}\right]$ such that our original affine coordinates are given by taking $x_{n+1}=1$, then $L_{0}$ is cut out by the equations $x_{1}=x_{2}=\cdots=x_{n}=0$, and $I(1)$ has global sections $x_{1}, \cdots, x_{n}$. A basis for the global sections of $I(d)$ consists of degree $d$ monomials in $x_{0}, \cdots, x_{n+1}$ having at least one factor from $x_{1}, \cdots, x_{n}$.

Case $s=1$. From the exact sheaf sequence on $P^{n+1}$,

$$
0 \rightarrow I \rightarrow O \rightarrow O_{0} \rightarrow 0,
$$

we twist by $d$ to get

$$
0 \rightarrow I(d) \rightarrow O(d) \rightarrow O_{0}(d) \rightarrow 0 .
$$

Since $L_{0}$ is a line in $P^{n+1}, O_{0} \otimes O(d) \cong O_{0}(d)$. For $d \geqslant-1, H^{i}\left(L_{0}, O_{0}(d)\right)=$ $H^{i}\left(P^{n+1}, O(d)\right)=0$ for $i \geqslant 1$. So the long exact sequence on cohomology implies that $H^{i}\left(P^{n+1}, I(d)\right)=0$ for $i \geqslant 2$, and thus the exact cohomology sequence collapses to

$$
0 \rightarrow H^{0}(P, I(d)) \rightarrow H^{0}(P, O(d)) \rightarrow H^{0}\left(L_{0}, O_{0}(d)\right) \rightarrow H^{1}(P, I(d)) \rightarrow 0,
$$

where, as above, $P=P^{n+1}$.

If $d=-1$, all of the $H^{0}$-groups above vanish, so $H^{1}(P, I(-1))=0$. For $d=0, H^{1}(P, I)=0$ by an earlier proposition. For $d \geqslant 1$, a basis of $H^{0}(P, O(d))$ consists of all monomials in $x_{0}, \cdots, x_{n+1}$ of degree $d$. A basis for $H^{0}(P, I(d))$ consists of all those monomials of degree $d$ containing at least one factor from $x_{1}, \cdots, x_{n}$. Thus a basis of $H^{0}(P, I(d))$ can be extended to a basis of $H^{0}(P, O(d))$ by including those monomials of degree $d$ in $x_{0}, x_{n+1}$ only, which is precisely a basis of $H^{0}\left(L_{0}, O_{0}(d)\right)$. Thus $h^{0}(P, O(d))=h^{0}(P, I(d))$ $+h^{0}\left(L_{0}, O_{0}(d)\right)$, and we conclude that $H^{1}(P, I(d))=0$.

Case $s \geqslant 2$. The proof now proceeds by induction on $s$. We assume the result for $s \geqslant 1$ and prove it for $s+1 \geqslant 2$. In particular, we assume $H^{i}\left(P, I^{s}(d)\right)=0$ for $d \geqslant s-2, i \geqslant 1$. Using the exact sheaf sequence

$$
0 \rightarrow I^{s+1} \rightarrow I^{s} \rightarrow \operatorname{Sym}^{s} N^{*} \rightarrow 0,
$$


we twist by $d \geqslant(s+1)-2=s-1$ to obtain

$$
0 \rightarrow I^{s+1}(d) \rightarrow I^{s}(d) \rightarrow\left(\operatorname{Sym}^{s} N^{*}\right)(d) \rightarrow 0 .
$$

Now $\operatorname{Sym}^{s} N^{*} \cong O_{0}^{\operatorname{rk}(s)}(-s)$, where $\operatorname{rk}(s)=\operatorname{rank}\left(\operatorname{Sym}^{s} N^{*}\right)=\left(\begin{array}{c}n-1+s \\ s\end{array}\right)$, so that $\left(\operatorname{Sym}^{s} N^{*}\right)(d) \cong O_{0}^{\operatorname{rk}(s)}(d-s)$. Because we assume $d \geqslant(s+1)-2=s-1$, $d-s \geqslant-1$, so that $H^{i}\left(L_{0}, O_{0}(d-s)\right)=0$ for $i \geqslant 1$. Then the long exact cohomology sequence obtained from $(* *)$ becomes

$$
\begin{aligned}
0 & \rightarrow H^{0}\left(P, I^{s+1}(d)\right) \rightarrow H^{0}\left(P, I^{s}(d)\right) \rightarrow H^{0}\left(L_{0},\left(\operatorname{Sym}^{s} N^{*}\right)(d)\right) \\
& \rightarrow H^{1}\left(P, I^{s+1}(d)\right) \rightarrow H^{1}\left(P, I^{s}(d)\right) \rightarrow 0,
\end{aligned}
$$

together with

$$
0 \rightarrow H^{i}\left(P, I^{s+1}(d)\right) \rightarrow H^{i}\left(P, I^{s}(d)\right) \rightarrow 0,
$$

for $i \geqslant 2$. By induction, $H^{i}\left(P, I^{s}(d)\right)=0, d \geqslant s-2, i \geqslant 1$, so that $H^{i}\left(P, I^{s+1}(d)\right)=0, i \geqslant 2, d \geqslant s-1$.

We now wish to count the dimension of $H^{0}\left(P, I^{r}(d)\right)$. A basis of $H^{0}\left(P, I^{r}(d)\right)$ consists of monomials of degree $d$ in $x_{0}, \cdots, x_{n+1}$ containing a factor of a monomial of degree $r$ in $x_{1}, \cdots, x_{n}$. To complete this to a basis of all of $H^{0}(P, O(d))$, we must include monomials of degree $d$ with fewer than $r$ factors from $x_{1}, \cdots, x_{n}$. The number of such monomials is equal to

$$
\sum\left(\begin{array}{c}
n-1+j \\
j
\end{array}\right)(1+d-j)
$$

where the summation is over $j=0, \cdots, r-1 .\left(\begin{array}{c}n-1+j \\ j\end{array}\right)$ is the number of monomials in $x_{1}, \cdots, x_{n}$ of degree $j$, while $1+d-j$ is the number of monomials in $x_{0}, x_{n+1}$ of degree $d-j$. Thus $h^{0}\left(P, I^{r}(d)\right)=h^{0}(P, O(d))-\Sigma(1+d$ $-j)\left(\begin{array}{c}n-1+j \\ j\end{array}\right)$. We apply this formula:

$$
h^{0}\left(P, I^{s}(d)\right)-h^{0}\left(P, I^{s+1}(d)\right)=(1+d-s)\left(\begin{array}{c}
n-1+s \\
s
\end{array}\right) .
$$

But this is precisely equal to $h^{0}\left(L_{0},\left(\operatorname{Sym}^{s} N^{*}\right)(d)\right)=\operatorname{rk}(s) \cdot h^{0}\left(L_{0}, O_{0}(d-s)\right)$. So the long exact cohomology sequence breaks up once more, and $H^{1}\left(P, I^{s+1}(d)\right) \cong H^{1}\left(P, I^{s}(d)\right)$, which vanishes by induction.

Using this proposition, we see that $H^{1}\left(P^{n+1}, I^{r+1}(d)\right)=0$, provided $d \geqslant r$ -1 . Since we have assumed $d \geqslant r$, there is no obstruction to lifting sections, and the proof of the sufficiency of the stated conditions is now complete.

Formula for $\gamma$. Assuming the conditions of the main theorem, we know of the existence of an algebraic hypersurface $\gamma$ which osculates our original $\gamma_{i}$ 's. A curious fact is that one can actually write down the degree $d$ polynomial defining $\gamma$. The proof that the $\gamma$ so defined actually osculates the original $\gamma_{i}$ 's is not a pleasant task and will not be taken up here. For details, see [2]. 
In homogeneous coordinates $\left[x_{0}, \cdots, x_{n+1}\right]$, chosen so that the affine coordinates used above are given by $x_{n+1}=1$, the polynomial of degree $d$ defining $\gamma$ is of the form

$$
p\left(x_{0}, \cdots, x_{n+1}\right)=\sum_{E}\left(\begin{array}{l}
d \\
E
\end{array}\right) B_{E} x^{E},
$$

where the summation is over all multi-indices $E=(E(0), E(1), \cdots, E(n+1))$ of degree $d$, i.e., $\sum_{i} E(i)=d$, and where $\left(\begin{array}{c}d \\ E\end{array}\right)=d ! /(E(0) ! \cdots E(n+1) !)$ and $x^{E}=x_{0}^{E(0)} \cdots x_{n+1}^{E(n+1)}$.

Let $S_{k}$ equal the $k$ th elementary symmetric polynomial in $\left\{X_{1}, \cdots, X_{d}\right\}$. Set $S_{0}=1$. Then

$$
B_{E}=(-1)^{d-E(0)} \frac{E(n+1) !}{\left(\begin{array}{c}
d \\
E(0)
\end{array}\right)(d-E(0)) !} \frac{\partial^{d-E(n+1)}\left[S_{d-E(0)}\right]}{\partial b_{1}^{E(1)} \cdots \partial b_{n}^{E(n)}},
$$

evaluated at $b=0$, gives the coefficients of $p$.

\section{References}

[1] P. Griffiths \& J. Harris, Principles of algebraic geometry, John Wiley, New York, 1978.

[2] J. A. Wood, An algebraization theorem for local hypersurfaces in projective space, Ph.D. Dissertation, University of California, Berkeley, 1982.

[3] _ A simple criterion for local hypersurfaces to be algebraic, preprint, 1983. 
